\title{
Alternate phase focusing in sequence of independent phased resonators as superconducting linac boosters
}

\author{
S. Dechoudhury* and Alok Chakrabarti \\ Variable Energy Cyclotron Centre, 1/AF Bidhan Nagar, Kolkata 700 064, India
}

(Received 30 May 2012; published 20 May 2013)

\begin{abstract}
Theoretical study of an alternate phase focusing (APF) structure, realized in a long chain of double gap quarter wave resonators, capable of accelerating heavy ions from 1.3 to $7 \mathrm{MeV} / \mathrm{u}$ has been carried out. Mathieu-Hill stability analysis for the focusing periods consisting of independent resonators with phase variation satisfying square-wave law has been used to evaluate parameters such as the electric field and phase for the resonators. Furthermore, a smooth approximation method taking into account the acceleration in the linac has been employed to find out the rf bucket parameters (energy and phase width acceptance) of the focusing periods. Corroborative particle tracking (longitudinal and transverse) has been carried out using simulated 3D fields for double gap quarter wave resonators (QWR). Steering effects in QWRs over the period have also been studied. In one APF period, the individual phase of resonators changes sign resulting in a vertical steering kick in a particular direction, which is less as compared to the case where all the individual resonators operate in the same phase.
\end{abstract}

DOI: 10.1103/PhysRevSTAB.16.052001

PACS numbers: 29.20.Ej, 29.27.-a

\section{INTRODUCTION}

The synchronous phase of a linear accelerator is chosen in the rising part of the waveform to ensure the longitudinal stability or bunching of the beam. But this selection also results in transverse defocusing for which additional transverse focusing elements such as solenoid/quadrupole magnets need to be provided. In an alternate phase focusing (APF) structure longitudinal and transverse beam focusing can be realized over a set of accelerating gaps by alternating the sign of the equilibrium phase at each gap. This principle was first proposed by Good and Fayenberg [1,2]. In a symmetrical APF (SAPF) structure having two accelerating gaps in a single focusing period $(\mathrm{Ng}=2$ denoting the number of accelerating gaps in a particular focusing period), phases are equal but opposite in sign $\left(\Phi_{1},-\Phi_{1}\right)$, while for asymmetrical APF (A-APF) phases are not equal but opposite in sign. In a modified APF structure the number of accelerating gaps in a period is more than two and the phase variation is of asymmetrical type. The disadvantage of the SAPF structure is that it has small longitudinal acceptance. On the other hand, longitudinal phase acceptance of the A-APF structure is appreciably large. Additionally, this structure offers simultaneous longitudinal and transverse focusing which is inherent to APF structures. The original idea of an asymmetrical alternating phase focusing (A-APF) has been proposed by Kushin almost 30 years ago $[3,4]$. Usually, the APF concept has

\footnotetext{
*sdc@vecc.gov.in
}

Published by the American Physical Society under the terms of the Creative Commons Attribution 3.0 License. Further distribution of this work must maintain attribution to the author(s) and the published article's title, journal citation, and DOI. been realized in multiacceleration gaps essentially in long drift tube structures. The number of gaps in a period over which the synchronous phase oscillates depends on the charge to mass $(q / A)$ ratio of the ions as well as with beam energy. The period usually contains about 10-20 accelerating gaps $(\mathrm{Ng})$. In the case of square-wave law, the synchronous phase alternates in a stepwise manner, where the set of neighboring gaps (say two or three in number) has the same synchronous phase. This stepwise sequence of neighboring gaps having the same phase can be realized in a sequence of independently phased resonators consisting of two or three gaps. Thus, it is possible to convert a long multigap resonator into a chain of short independently phased resonators. Studies have also established that the chain of such short independent resonators, consisting of a fixed number of gaps in each resonator, can be as effective as a long multigap resonator [5-7]. Minaev [6] has considered resonators with one or two gaps, while four gaps structures have been used by Masunov [7]. The total number of gaps in one such focusing period $(\mathrm{Ng})$ in fact needs to be increased with beam energy in order to preserve the position of the working point on the Mathieu-Hill stability diagram. A recent feasibility study by Kapin et al., [8] also demonstrated the effectiveness of this concept. They have evaluated the design parameters for the $0.5-6.0 \mathrm{MeV} / \mathrm{u}$ heavy-ion linac with charge to mass ratio of $1 / 8$ using only the stability diagram analysis. As compared to studies carried out earlier [6,7], this concept requires a less number of independent resonators to have the desired energy gain. In principle, one can effectively minimize the number of resonators keeping the phase variation flexible.

At Variable Energy Cyclotron Centre, as an extension of present Isotope Separator On Line (ISOL)-type rare ion beam (RIB) facility [9], a new proposal for building up an 
advanced RIB facility (ANURIB) has been placed before the scientific and funding committee [10]. The unique features of this facility are: (i) two primary accelerators giving a low cost option for producing both neutron-rich and proton-rich RIBs, acceleration up to $7 \mathrm{MeV} / \mathrm{u}$ in $\mathrm{HI}$ linac and further acceleration to $100 \mathrm{MeV} / \mathrm{u}$ in the separated sector cyclotron, (ii) fragmentation of pure secondary $n$-rich RI beams enabling a unique opportunity to produce dripline nuclei, (iii) both ISOL and Projectile Fragment Separator-type facility, (iv) acceleration of both rare and $\beta$ stable ion beams. The RIB of interest will be selected in an isotope separator and accelerated to $1.3 \mathrm{MeV} / \mathrm{u}$, first in a radio frequency quadrupole linear accelerator, followed by a number of interdigital $H$-type cavity linacs. This energy will be further enhanced to $\sim 7 \mathrm{MeV} / \mathrm{u}$ in superconducting quarter wave resonator (QWR) linac boosters.

The present paper addresses the design of heavy-ion linac boosters in A-APF configurations using double gap QWR's as independent resonators. The input and the output energy for this design have been chosen to be $1.3 \mathrm{MeV} / \mathrm{u}$ and $7 \mathrm{MeV} / \mathrm{u}$, respectively, for a minimum $q / A$ of $1 / 8$. Stability analysis has been carried out to evaluate the parameters of the focusing periods. Selection of number of gaps in each QWR and designed beta has been dictated by the need of having appreciable transit time factor (TTF) over the mentioned energy range. Smooth approximation taking into account the acceleration in SC linacs has been used to calculate the rf bucket parameters. 3D fields of QWR have been simulated using CST MICROWAVE STUDIO [11]. Particle tracking using GPT [12] has been performed with the 3D fields for the entire energy range from 1.3 to $\sim 7 \mathrm{MeV} / \mathrm{u}$. Steering effects have also been calculated for the chosen A-APF configuration.

\section{DESIGN OF A-APF CONFIGURATION}

\section{A. Selection of designed beta $\left(\beta_{d}\right)$ for QWR}

The designed beta $\left(\beta_{d}\right)$ for QWR's is selected on the basis of transit time factor over the range of 1.3 to $7 \mathrm{MeV} / \mathrm{u}$. The transit time factor (TTF) of a double gap

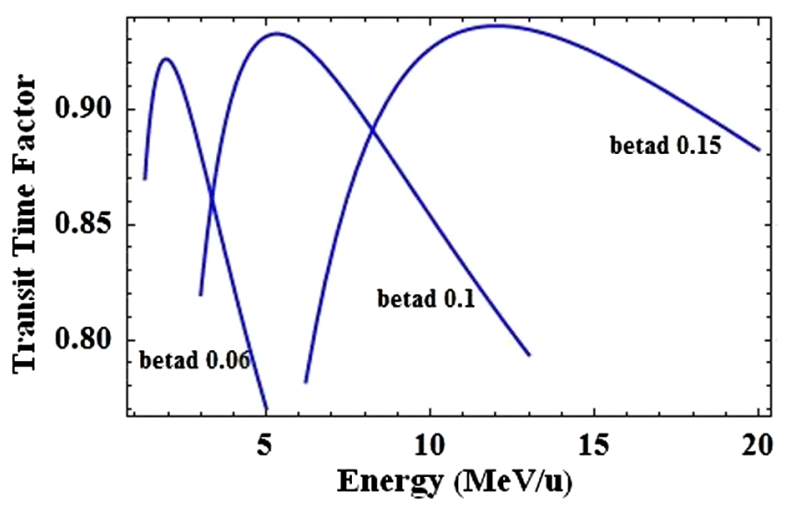

FIG. 1. Transit time factor for double gap quarter wave resonator with designed beta $0.06,0.1$, and 0.15 .
QWR can be calculated using the formula described in [13]. We have chosen frequency to be $100 \mathrm{MHz}$, aperture diameter $20 \mathrm{~mm}$, and gap to $\beta_{d} \lambda$ ratio as 0.2 . The choice of beta of the resonators $\left(\beta_{d}\right)$ as well as the number of resonators in a period should take care of the fact that TTF remains greater than 0.8 for the entire energy range and for all the different masses. $q / A=1 / 8$ seems to be a judicious choice. As an example, charge stripping at the energy of $1.3 \mathrm{MeV} / \mathrm{u}$ would produce average charge state of $36^{+}$for ${ }^{238} \mathrm{U}$, while $q / A=1 / 8$ corresponds to charge state of $30^{+}$. For $q / A=1 / 8$ the desired energy gain is $5.7 \mathrm{MeV} / \mathrm{u}$ with input beam of $1.3 \mathrm{MeV} / \mathrm{u}$. In the case of ${ }^{16} \mathrm{O}$, the average charge state after the stripper at $1.3 \mathrm{MeV} / \mathrm{u}$ is $6^{+}$resulting in an approximate final energy of $18 \mathrm{MeV} / \mathrm{u}$ for the same energy gain per unit charge. The calculated TTF for the energy range of $1.3-18 \mathrm{MeV} / \mathrm{u}$ is shown in Fig. 1.

\section{B. Stability analysis}

In square-wave law, as described before, the set of neighboring gaps has a constant synchronous phase. Each of such gaps can be transformed into a single independent resonator operating at the particular phase. In this way, one can easily transform the stepwise phase variation of the gaps into a chain of independent resonators. The A-APF configuration provides both transverse and longitudinal focusing over the period, but the focusing strength decreases with increase in particle velocity. Therefore, in a particular focusing period we need to increase the number of gaps with energy. In a particular focusing period, the electric field is kept constant, while only the phase is varied. A previous study [8] has stated that with these features, one can minimize the number of independent resonators to achieve the desired energy gain. We would

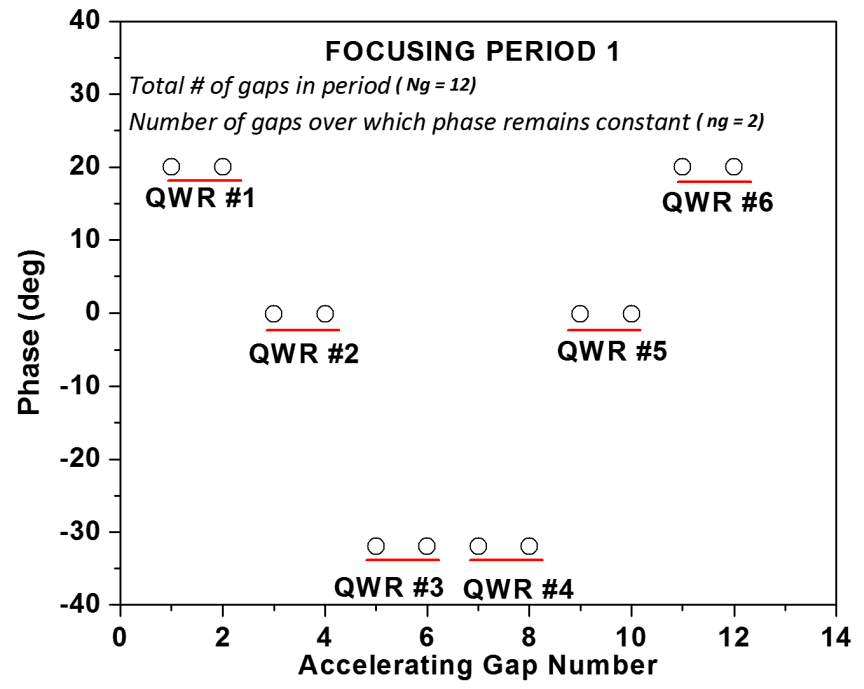

FIG. 2. Square-wave law variation for first focusing period with total number of accelerating gaps $\left(N_{g}=12\right)$ with phase constant for two gaps corresponding to single QWR $\left(n_{g}=2\right)$. 

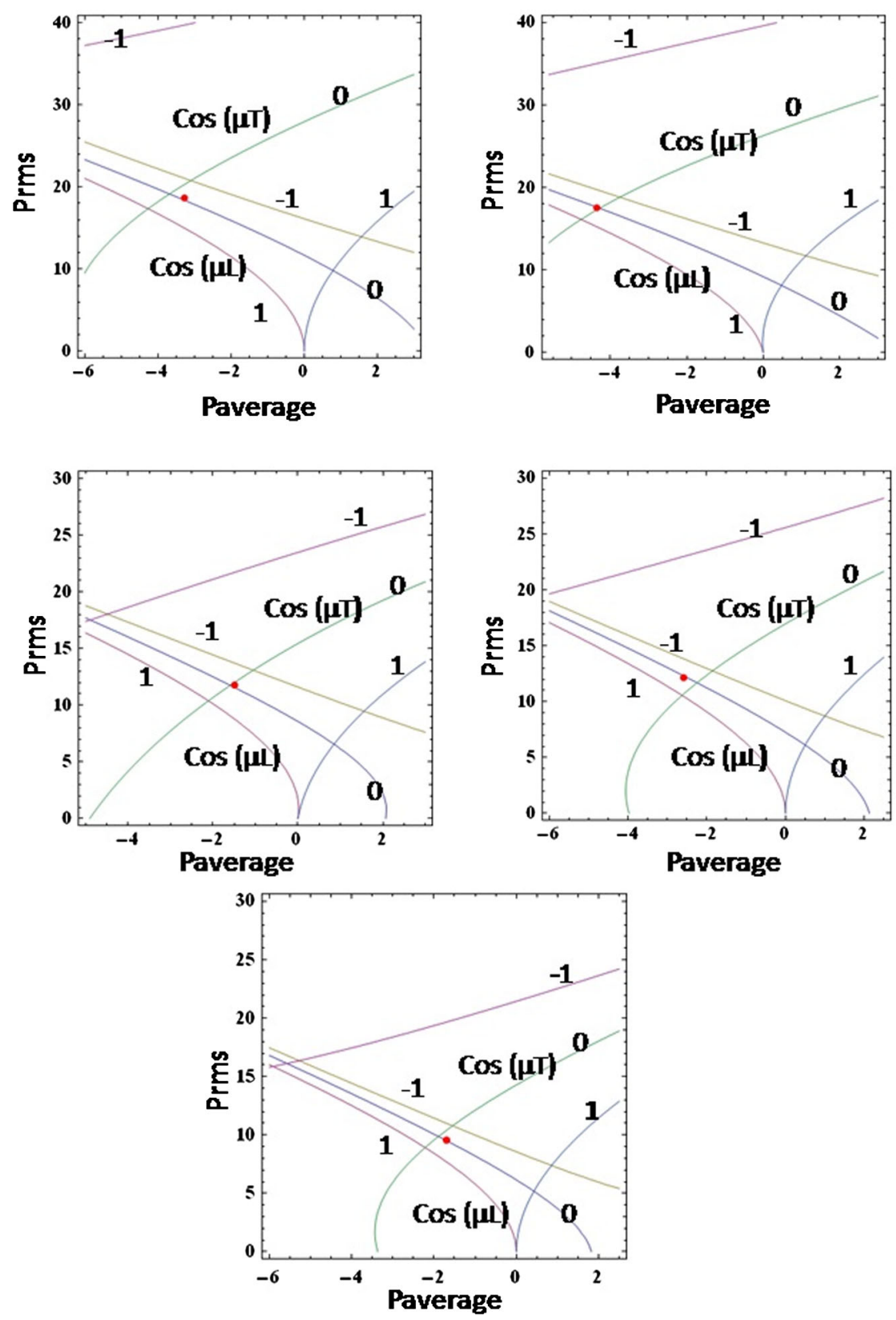

FIG. 3. Neck-tie stability diagram for five focusing periods, with red points showing the position of operating points.

like to utilize these advantages of stepwise phase variations for our design.

The synchronous phase oscillation over a single period was chosen to follow the stepwise function as described in [14] obeying the relation $\varphi_{s}(\tau)=\bar{\varphi}+\tilde{\varphi}(\tau)$, where $\tau=$ $z / L_{f} ; L_{f}$ being the focusing period length over which the phase excursion completes one cycle and $z$ being the longitudinal coordinate. The phase variation over a focusing period consists of a constant part $\bar{\varphi}$ and a variable part $\tilde{\varphi}(\tau)$. The variable part remains constant in the neighboring gaps leading to a constant synchronous phase for those gaps. In the case of a focusing period consisting of double gap QWRs as the independent phase resonators, phases are the same for the two gaps $\left(n_{g}\right.$; see Fig. 2) of a QWR. Figure 2 shows the first focusing period consisting of six QWR resonators, with $N_{g}=12$ and $n_{g}=2$. In terms of phase deviation from the synchronous particle $\Psi=$ $\phi-\phi s, \phi s$ being the phase of the synchronous particle, and dimensionless radial position $\rho=r / L_{f}$, the MathieuHill equations [14] take the form:

$$
\begin{aligned}
d^{2} \psi / d \tau^{2}+P_{\psi}(\tau) \psi & =0 \quad \text { and } \\
d^{2} \rho / d \tau^{2}+P_{\rho}(\tau) \rho & =0,
\end{aligned}
$$

where 
TABLE I. Final parameters for all five focusing periods.

\begin{tabular}{|c|c|c|c|c|c|}
\hline Focusing period number & 1 & 2 & 3 & 4 & 5 \\
\hline Energy (input-output) $\mathrm{MeV} / \mathrm{u}$ & $1.3-2.06$ & $2.06-2.78$ & $2.78-3.84$ & $3.84-5.08$ & $5.08-7.16$ \\
\hline Length $(\mathrm{m})$ & 2.1 & 2.4 & 3.24 & 3.66 & 4.86 \\
\hline Number of $\mathrm{QWR} / \beta$ designed & $6 / 0.06$ & $7 / 0.06$ & $7 / 0.1$ & $8 / 0.1$ & $8 / 0.15$ \\
\hline Phase acceptance (deg) & 131 & 158 & 117 & 124 & 128 \\
\hline Energy width $(\mathrm{keV} / \mathrm{u})$ & 227 & 338 & 353 & 444 & 631 \\
\hline
\end{tabular}

$$
\begin{aligned}
P_{\psi}(\tau) & =2 B \sin [\bar{\varphi}+\tilde{\varphi}(\tau)] \quad \text { and } \\
P_{\rho}(\tau) & =-B \sin [\bar{\varphi}+\tilde{\varphi}(\tau)+\psi],
\end{aligned}
$$

which follow the properties of a stepwise function, namely, $P_{\psi}(\tau) \equiv P_{\psi}(\tau+1)$ and $P_{\rho}(\tau) \equiv P_{\rho}(\tau+1)$.

In the equation above, $B$, the focusing strength, is given by $B=\left(\pi q E_{m} / A m_{0} c^{2}\right)\left(L_{f} / \beta_{s} \lambda\right)^{2}\left(1-\beta_{s}^{2}\right)^{3 / 2}, E_{m}$ is the product of the average electric field and the transit time factor, $q / A$ is the charge to mass ratio, and $\beta_{s}$ us the synchronous velocity. To solve the above Mathieu-Hill equations, a matrix multiplication technique has been used with every rf gap treated as a single thin lens. In order to analyze the solutions, one creates the well-known SmithGluckstern stability diagram [15].

For a particular period, consisting of say $N$ number of double gap QWRs (which means number of accelerating gaps in the period $N g=2 N$ ), different sets of phases for the QWRs are chosen, obeying the relation $\bar{\varphi}+\varphi_{0} \sin [\tau]$ by varying $\bar{\varphi}$ and $\varphi_{0}$. The electric field for a particular set of such phases has been kept the same for all the QWR's. The rms and average values of $P_{\psi}(\tau)$ and $P_{\rho}(\tau)$ have been calculated for each such set of phase and electric field. The maximum electric field gradient (product of electric field

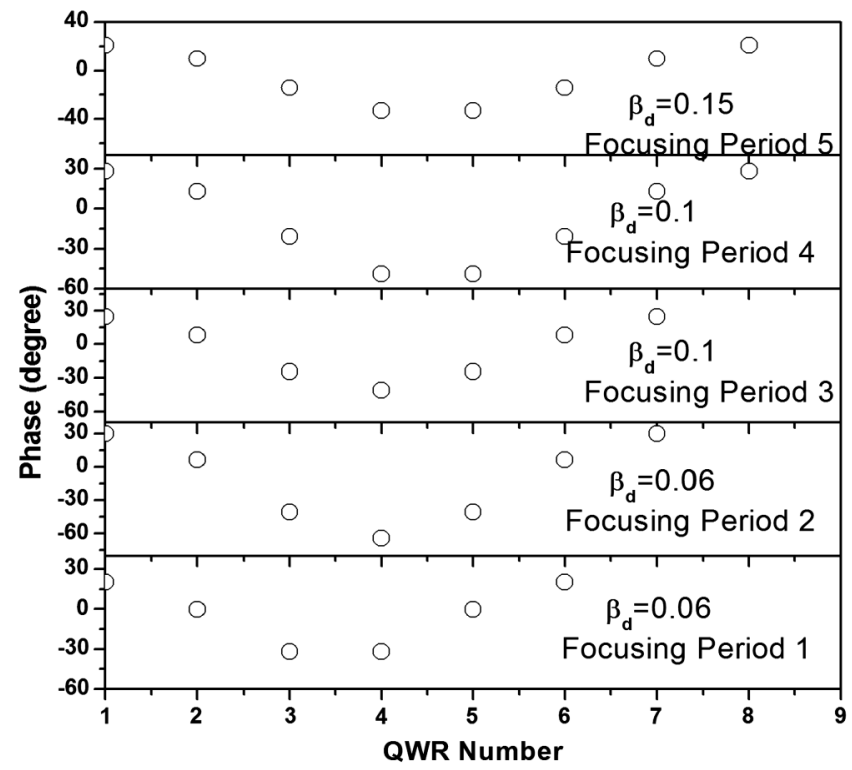

FIG. 4. Synchronous phase variation for the QWR in a particular focusing period. and TTF) has been taken to be $6.5 \mathrm{MV} / \mathrm{m}$. For a particular focusing period and choosing a particular set of phase and electric field, matrices are obtained by multiplying in proper order the matrices of drift lengths and electric field gaps. The matrices have been calculated numerically using MATHEMATICA [16]. In a focusing period, appreciable distance in between QWR's has been kept and also space has been provided to accommodate a solenoid for additional transverse focusing. Corresponding to each such set of phase and electric field, representing a point in phase space created by rms and average value of $P_{\psi}(\tau)$ and $P_{\rho}(\tau)$, transverse $\left(\mu_{T}\right)$ and longitudinal phase advance $\left(\mu_{L}\right)$ have been calculated using the matrix multiplication technique. It is well known that cosine of phase advances in both directions should have modulus value less than 1 for
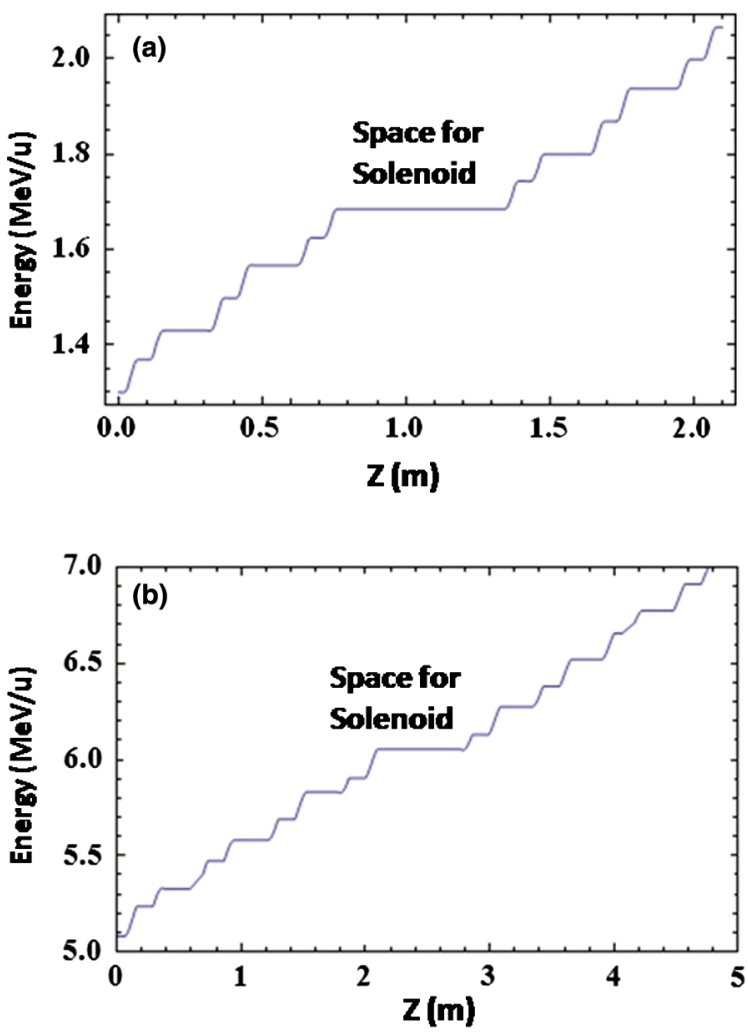

FIG. 5. Reference particle energy along $z$ for (a) first focusing period consisting of six QWRs with gap for solenoid in between, and (b) last focusing period consisting of eight QWRs with gap for solenoid after fourth QWR. 


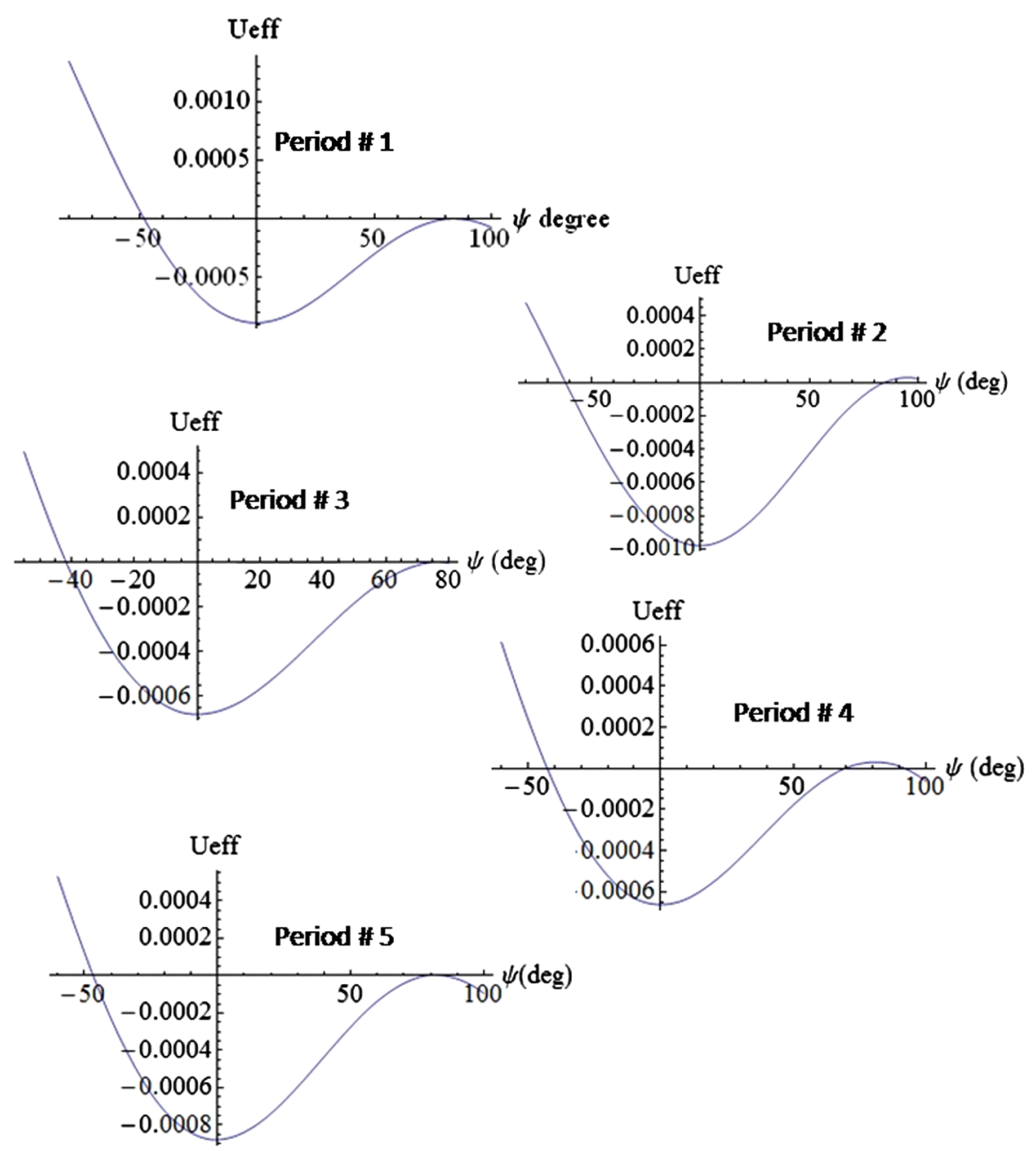

FIG. 6. Effective potential as a function of phase for all focusing periods using smooth approximation with acceleration.

stability. Contours of $\cos \left(\mu_{L}\right)$ and $\cos \left(\mu_{T}\right)$ having values 1,0 , and -1 are drawn on the phase space created by rms and average values of $P_{\psi}(\tau)$ and $P_{\rho}(\tau)$. In order to ensure strong focusing in the longitudinal and transverse directions, the working point is chosen at the intersection of contour lines $\cos \left(\mu_{L}\right)=0$ and $\cos \left(\mu_{T}\right)=0$.

\section{Result of stability analysis}

The optimized design consists of five such focusing periods. We have designed the first two periods consisting of QWRs with $\beta_{d}=0.06$, the next two focusing periods with $\beta_{d}=0.1$, and the last one consisting of resonators with $\beta_{d}=0.15$. Final energy after five such focusing periods is $7.16 \mathrm{MeV} / \mathrm{u}$ for $q / A=1 / 8$. Each focusing period can be housed in a separate cryomodule. So we have altogether five cryomodules, parameters of which are listed in Table I. The number of QWRs of particular $\beta_{d}$ in a focusing period is increased with energy to ensure that the operating point lies in the center of the stability diagram for all the focusing periods. The stability diagrams for five focusing periods are shown in Fig. 3 and selected phase variation of each focusing period in Fig. 4.

\section{Calculation of rf bucket parameters of the focusing periods}

To calculate the longitudinal phase acceptance and energy width, a smooth approximation without acceleration has been carried out before [17]. However, in the case of a superconducting linac since the energy gain is quite appreciable, acceleration effects need to be invoked. Therefore we have adopted smooth approximation formalism with acceleration [18]. The equation of motion in the longitudinal dimension neglecting the space charge forces is given by [18]

$$
\begin{aligned}
\psi^{\prime \prime} & -\gamma_{0}^{3} \beta_{0}^{3}\left(1 / \gamma_{0}^{3} \beta_{0}^{3}\right)^{\prime} \psi^{\prime}-(\omega / c)\left(1 / \gamma_{0}^{3} \beta_{0}^{3}\right) \\
& \times\left(q / A m c^{2}\right) \sum_{i} E_{0 i}(z)\left\{\cos \left[\omega t_{0}(z)+\theta_{i}\right]\right. \\
& \left.-\cos \left[\omega t_{0}(z)+\theta_{i}+\psi\right]\right\}=0 .
\end{aligned}
$$

In the above equation $E_{0 i}$ is the field amplitude and $\theta_{i}$ is driven phase for the $i$ th cavity, $\psi=\omega\left(t-t_{0}\right)$ is the phase relative to reference phase, the prime denoting differentiation with respect to longitudinal coordinate $z$. A summation has been done for all the resonators; $q / A$ is charge to mass ratio. $\beta_{0}$ denotes velocity of the reference particle. 


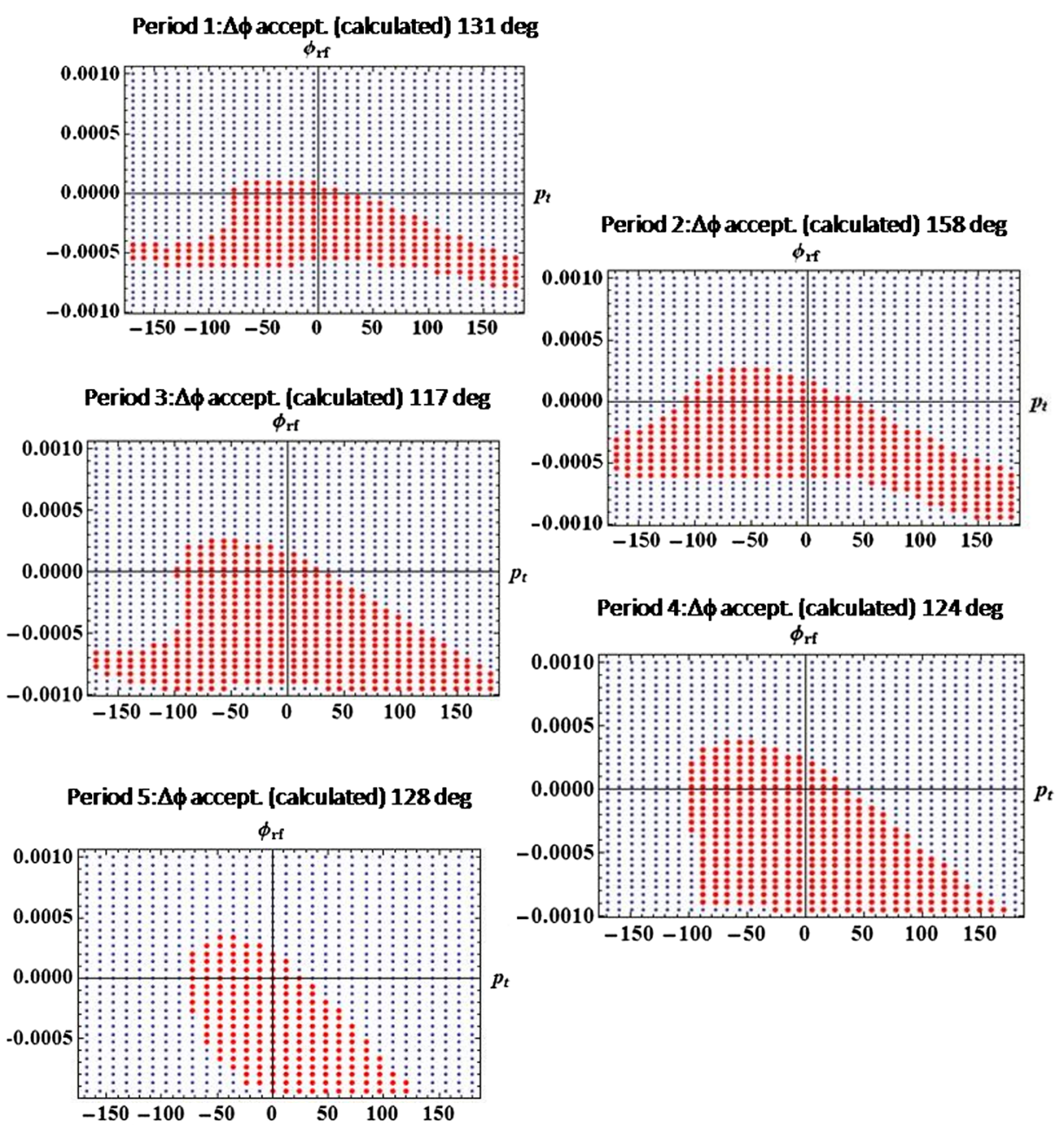

FIG. 7. Longitudinal acceptance of the periods evaluated by solving the longitudinal equation of motion.

Defining a new variable $\phi=\sqrt{\gamma_{0}^{3} \beta_{0}^{3}} \psi$, the equation of motion would be $\phi^{\prime \prime}=F(\phi, z)$, where

$$
\begin{aligned}
F(\phi, z)= & \left(\frac{1}{2} a^{\prime}(z)+\frac{1}{4} a^{2}(z)\right) \phi+\frac{\omega}{c} \frac{1}{\sqrt{\gamma_{0}^{3} \beta_{0}^{3}}} \\
& \times \frac{q}{A m c^{2}} \sum_{i} E_{0 i}(z)\left\{\cos \left[\omega t_{0}(z)+\theta_{i}\right]\right. \\
& \left.-\cos \left[\omega t_{0}(z)+\theta_{i}+\phi / \sqrt{\gamma_{0}^{3} \beta_{0}^{3}}\right]\right\},
\end{aligned}
$$

where $a(z)=3 \gamma_{0}^{\prime} / \gamma_{0} \beta_{0}^{3}$.

Since we are considering acceleration, $\gamma_{0}$ and $\beta_{0}$ are functions of $z$. It is assumed that particle phase is the sum of a fast oscillating part and smooth slow variation. Therefore the net force can be separated into two parts: one for fast oscillation $(\tilde{\phi})$ and the other for slow variation $(\bar{\phi})$. The equation of motion for slow variable can be rewritten as $\bar{\phi}^{\prime \prime}=\bar{F}(\bar{\phi})+f(\bar{\phi})$, where the second term is the ponderomotive force. The effective potential can be written as

$$
U_{\text {eff }}(\bar{\phi})=-\int_{0}^{\bar{\phi}} d x[\bar{F}(x)+f(x)]
$$

From the effective potential, one can calculate the phase acceptance and energy width simultaneously [17].

In order to evaluate the longitudinal parameters (energy and phase) of the reference particle in a particular focusing period, the equation of motion is solved with the electric field and phases of the cavities as determined from a stability diagram. The relevant set of differential equations solved in order to ascertain the reference particle energy is given by

$$
\begin{aligned}
\gamma^{\prime}(z) & =\frac{q}{A m c^{2}} \sum_{i} E_{0 i}(z)\left\{\cos \left[\omega t_{0}(z)+\theta_{i}\right]\right\}, \\
t^{\prime}(z) & \left.=1 /\left\{c \sqrt{\left[1-\gamma^{-2}(z)\right.}\right]\right\} .
\end{aligned}
$$

Reference particle energy with longitudinal coordinate $z$ for the first and fifth focusing period is shown in Fig. 5. The onaxis longitudinal electric field as simulated by CST has been used for this calculation and has been presented in Sec. III. Using MATHEMATICA, the effective potential for all five 
focusing periods has been calculated with steps described above. The plots of effective potential as a function of phase using smooth approximation for all the periods are shown in Fig. 6. The phase acceptance and the energy width calculated from effective potential as mentioned in [17] have been enlisted in Table I. Solving the longitudinal equation of motion as described by the set of equations for $t[z]$ and $\gamma[z]$ using different initial conditions of $t[0]$ and $\gamma[0]$, one can numerically determine the longitudinal acceptance of each focusing periods. The results are shown in Fig. 7, with blue dots showing the initial set for $p_{t}=\gamma-\gamma_{0}$ and $\Psi$ while red dots indicating those having final $\left|p_{t} / \gamma_{0}\right|<0.001$. Defining phase acceptance as width $\Delta \varphi$ on the $p_{t}=0$ axis [18], one can see that simulated $\Delta \phi$ agrees well with that derived from the effective potential as shown in Fig. 6.

\section{BEAM DYNAMICS SIMULATION}

\section{A. rf simulation of quarter wave resonators}

A CST simulation of quarter wave resonators has been carried out to have the 3D field distribution of the cavities. The resonant frequency for all the resonators, drift tube diameter and gap to $\beta_{d} \lambda$ ratio for all the cavities are taken to be $100 \mathrm{MHz}, 20 \mathrm{~mm}$, and 0.2 , respectively. In case of superconducting cavities, the resonant structure needs to be optimized so as to minimize the peak magnetic and electric fields at the required acceleration gradients (not exceeding $6.5 \mathrm{MV} / \mathrm{m}$ ). The resonators are cylindrical at the bottom and conical at the top, similar to the structure described in [19]. Different geometrical parameters and their dependence on peak electric and magnetic field as mentioned in Ref. [19] formed the basis of our structure optimization. The structures for the designed beta of $0.06,0.1$, and 0.15 have been shown in Fig. 8. In order to reduce the steering effect, (described later) tilt angle has been introduced for QWR with $\beta_{d}=0.15$. The longitudinal electric field along the axis has also been plotted in Fig. 8. The final design parameters of QWR are given in Table II.

\section{B. Particle tracking simulation}

The particle tracking has been carried out using the GPT with the 3D fields of the three types of quarter wave resonators as obtained from CST simulation. We are accelerating RIBs, where the beam intensity is never high enough for space charge to be considered. So, we have used GPT tracking without the space charge. The correct phase and voltage multiplication factor for each of the QWR fields in GPT are the same as that calculated during numerical analysis described before. Using GPT, the phase acceptance of each focusing period has been analyzed in order to compare the same with previous numerical calculation. Retracing back those particles that have final values of $\left|p_{t} / \gamma_{0}\right|<0.001$, the phase acceptance has been calculated as shown in Fig. 9. For the first four focusing periods, they are in good agreement with numerical calculation. In
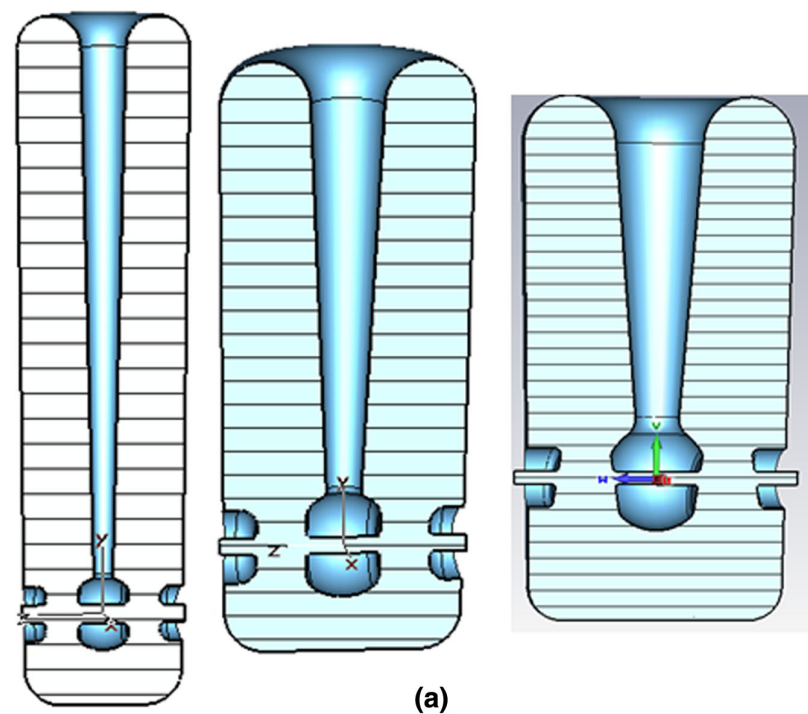

(a)

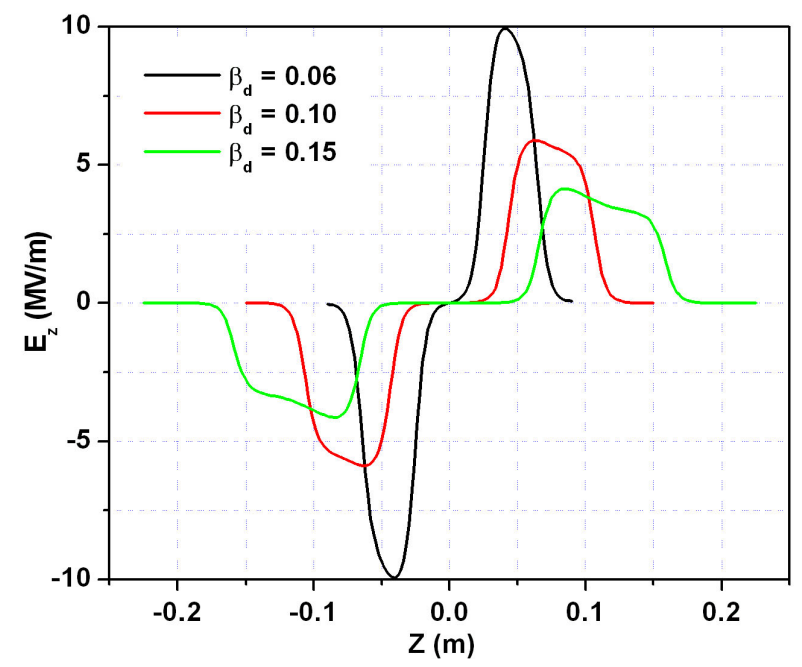

(b)

FIG. 8. (a) QWR cavities with $\beta d 0.06,0.1$, and 0.15 , and (b) CST simulated on-axis electric field for three resonators.

the case of QWR with $\beta_{d}=0.15$ (used in fifth focusing period), the phase acceptance area has been found to be less than the value predicted by the numerical analysis. The particle tracking shows that steering reduces the phase acceptance. So, QWR with $\beta_{d}=0.15$ has been remodeled using CST with $9^{\circ}$ tilt angle both in beam ports and drift tubes. The new distribution of fields thus generated has been used both in analytical and GPT calculation. The result

TABLE II. Design parameters of QWR simulated by CST.

\begin{tabular}{lccc}
\hline \hline Designed beta $(\beta d)$ & 0.06 & 0.10 & 0.15 \\
Epeak @ $1 \mathrm{MV} / \mathrm{m} \mathrm{Ea}$ & $4.5 \mathrm{MV} / \mathrm{m}$ & $4.12 \mathrm{MV} / \mathrm{m}$ & $5 \mathrm{MV} / \mathrm{m}$ \\
Bpeak @ $1 \mathrm{MV} / \mathrm{m} \mathrm{Ea}$ & $76.6 \mathrm{Gs}$ & $80 \mathrm{Gs}$ & $94 \mathrm{Gs}$ \\
Stored energy $(U)$ & $0.032 \mathrm{~J}$ & $0.102 \mathrm{~J}$ & $0.258 \mathrm{~J}$ \\
$@ 1 \mathrm{MV} / \mathrm{m} \mathrm{Ea}$ & & & \\
$R / Q$ & 724 & 575 & 617 \\
Voltage gain @ 1 J & $0.69 \mathrm{MV}$ & $0.66 \mathrm{MV}$ & $0.62 \mathrm{MV}$ \\
\hline \hline
\end{tabular}




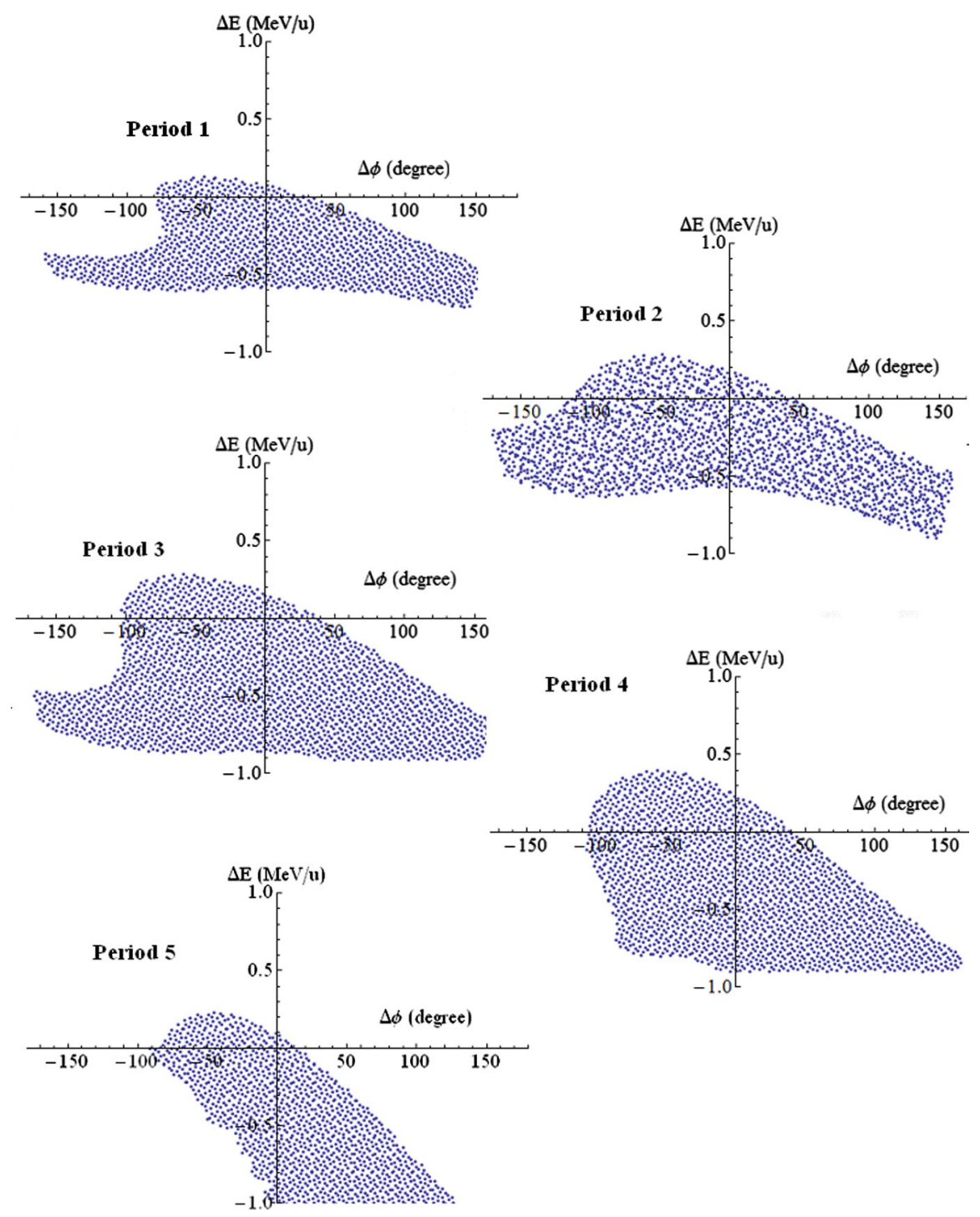

FIG. 9. Longitudinal acceptance of the periods using particle tracking code GPT.

of GPT tracking vis-à-vis phase acceptance is shown in Fig. 9. The results are in agreement with the numerical analysis. In order to bring out the efficacy of the alternate phase focusing concept, a GPT run for the first focusing period with parallel beam ranging from $-10 \mathrm{~mm}$ to $+10 \mathrm{~mm}$ without any focusing element/solenoid is shown in Fig. 10.

\section{Steering effect analysis}

In quarter wave resonators, steering is caused by the vertical $(y)$ component of the electric field and the horizontal $(x)$ component of the magnetic field. The steering depends on the resonator's synchronous phase, beam velocity, and acceleration gradient. In superconducting structures steering is more pronounced due to very high field gradient. This effect was first pointed out in 2001 [20]. Steering due to magnetic force is always 1 order of magnitude higher than that due to electric field. Steering, being proportional to the sinusoidal of the synchronous phase is maximum at bunching and disappears at $0^{\circ}$. The net steering component increases with cavity aspect ratio (horizontal/vertical dimensions). To correct for the steering, two remedies were proposed earlier [21]. The first one is offaxis injection of beam, thus creating the rf defocusing/ focusing to counteract the transverse electric and magnetic steering. This is valid for a wide range of beam velocity $(\beta)$, but since rf defocusing is inversely proportional to $\beta^{3}$, in case of higher $\beta$, vertical offset required is comparable to aperture of drift tube. The second one is beam port tilting which results in an effective $E y$ of either positive/negative sign to counteract the kick. It is useful for acceleration in the velocity range from $0.7 \beta_{d}$ to $2 \beta_{d}$. This remains effective until the $E y$ correction component required is small compared to the $E z$ field. The offset correction can reduce the steering effect in case of beam velocity up to $\beta \sim 0.1$, while beam port tilting can extend this range to about $\beta \sim 0.15$. Apart from this since magnetic field causes more steering, any shape modifications of drift tube or 


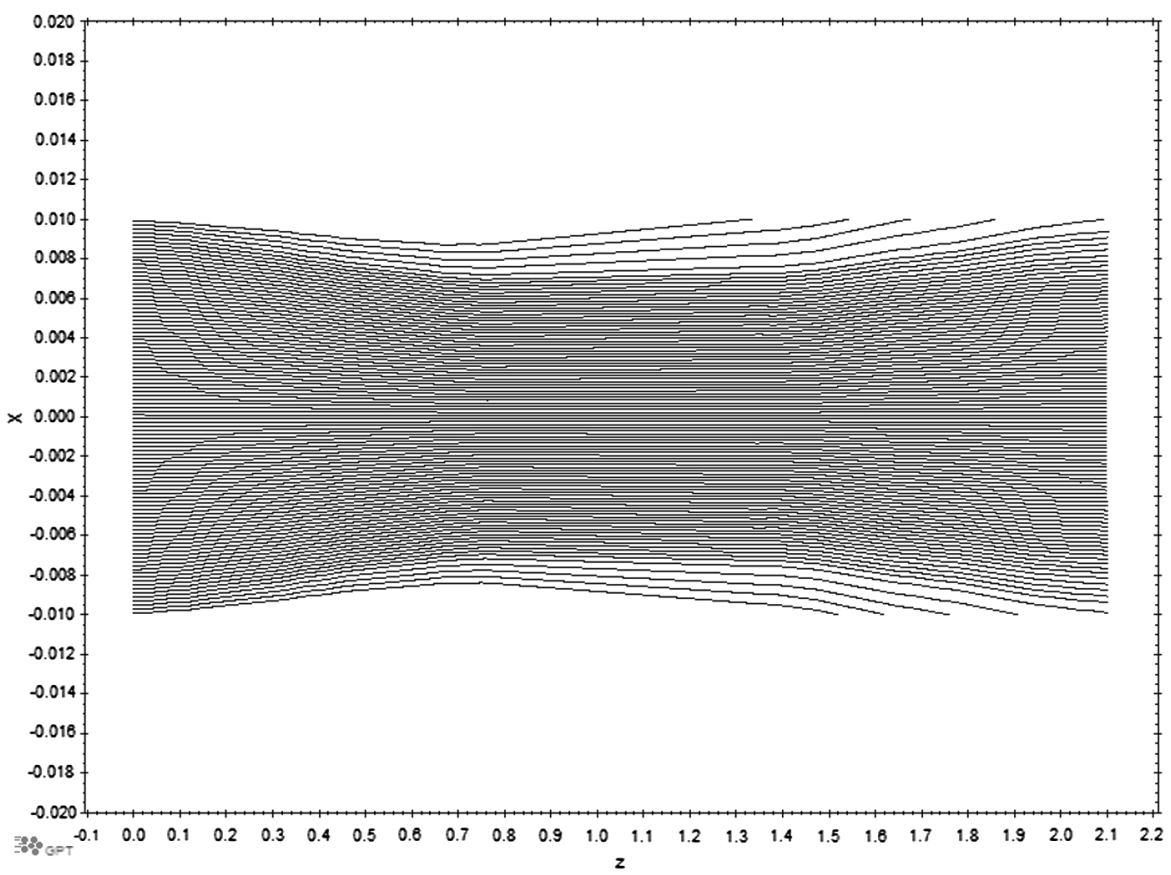

FIG. 10. Trajectory of parallel beam through first focusing period.

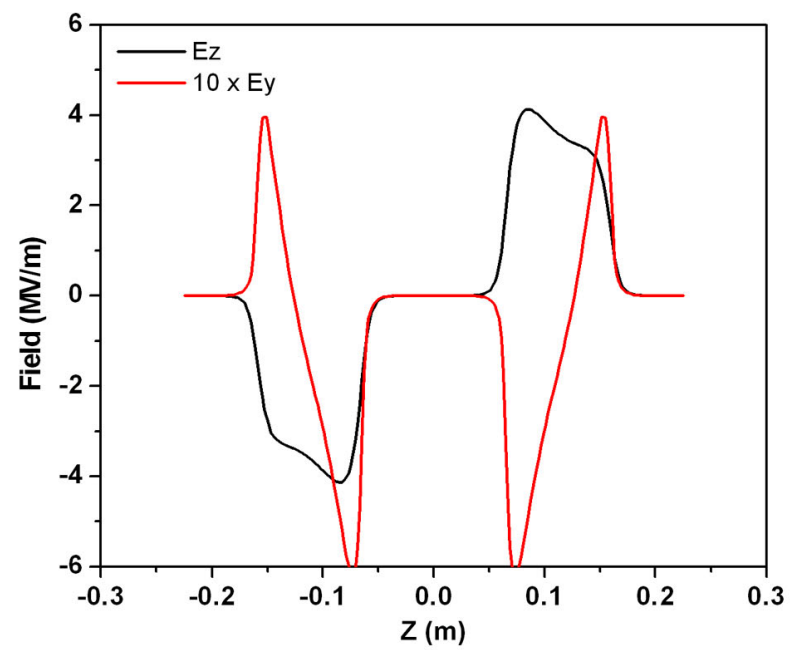

(a)
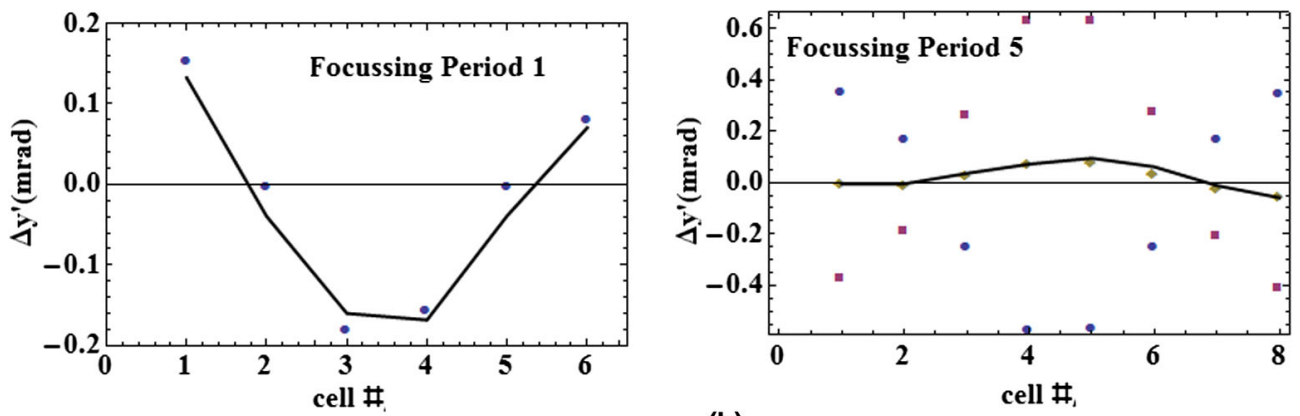

(b)

FIG. 11. (a) Electric field for beta $=0.15$ QWR with tilt angle of $9^{\circ}$. (b) Steering kick calculated using an analytical expression while the black line shows the GPT simulation. Blue dots in period \#1 show net steering. In the case of period \#5, magenta shows the electric part, blue shows the magnetic part, while yellow dots are net effect. 
the inner conductor which reduces the magnetic field in beam drift region would help in minimizing the steering effect. In our case, QWRs are of reduced diameter type (both inner and outer conductor), which reduces the effective magnetic field in the beam drift region. In a particular focusing period, the phase oscillates from positive value to negative value thus reducing the effective kick in any one direction. Therefore, no special efforts have been made to take care of the steering in the chain of cavities (28 in numbers) contained in the first four periods. However, for the last period comprised of QWRs with $\beta_{d} \sim 0.15$, the steering effect needs to be properly taken into account. This is done by introducing a tilt angle in the beam port and drift tube which reduces the net steering kick. Longitudinal acceptance for the fifth focusing period, calculated using
GPT with the remodeled real 3D field are in agreement with that calculated analytically.

In a recent paper on steering, the authors of [22] have reported analytical expressions of steering due to electric and magnetic field, which can be calculated from geometrical field values. The steering calculated using these analytical expressions is found to be in good agreement with particle tracking results. The two important geometrical parameters pertaining to magnetic and electric field steering are given by the following expressions:

$$
G B x=\frac{c \int_{0}^{L / 2} B x(z) d z}{\int_{0}^{L / 2} E z(z) d z} \text { and } G E y=\frac{\int_{0}^{L / 2} E y(z) d z}{\int_{0}^{L / 2} E z(z) d z},
$$

while the angular kick is given by the formula

$$
\Delta y /(E a, \phi, \beta)=\frac{q E a L \sin \phi}{A m c^{2} T_{E z}\left(\beta_{0}\right)}\left\{\frac{G B x}{\beta \gamma} T_{g}\left(\beta, g_{B x}\right) \sin \left(\frac{\pi d_{b x}}{\beta \lambda}\right)+\frac{G E y}{\beta^{2} \gamma} T_{g}\left(\beta, g_{E y}\right) \cos \left(\frac{\pi d_{E y}}{\beta \lambda}\right)\right\},
$$

where $L$ is length of cavity, $E a$ is acceleration gradient, $\varphi$ is synchronous phase, while the transit time factors $(T)$, effective gap length $(g)$, and gap to gap distance $(d)$ are defined as [22]

$$
\begin{gathered}
T_{g}\left(\beta, g_{B x}\right)=\frac{\sin \left(\pi g_{B x} / \beta \lambda\right)}{\left(\pi g_{B x} / \beta \lambda\right)}, \quad T_{g}\left(\beta, g_{E y}\right)=\frac{\sin \left(\pi g_{E y} / \beta \lambda\right)}{\left(\pi g_{E y} / \beta \lambda\right)} \quad d_{E y}=2 \frac{\int_{0}^{L / 2} z E_{y}(z) d z}{\int_{0}^{L / 2} E_{y}(z) d z}, \quad d_{B x}=2 \frac{\int_{0}^{L / 2} z B_{x}(z) d z}{\int_{0}^{L / 2} B_{x}(z) d z} \\
g_{E y}=\frac{4 \int_{0}^{L / 2}\left|z-\left(d_{E y} / 2\right)\right| E_{y}(z) d z}{\int_{0}^{L / 2} E_{y}(z) d z}, \quad g_{B x}=\frac{4 \int_{0}^{L / 2}\left|z-\left(d_{B x} / 2\right)\right| B_{x}(z) d z}{\int_{0}^{L / 2} B_{x}(z) d z} .
\end{gathered}
$$

These modified values can be evaluated once the magnetic and electric field profiles are known. In case $y \ll R$, one can evaluate the kick using the on-axis field profile. Using CST simulated field profiles, the vertical kick has been calculated and compared with particle tracking code GPT. For QWR with designed beta 0.15 , the tilt angle of $9^{\circ}$ would provide an additional field $E y$, which acts in the opposite direction of the kick introduced by the magnetic field, thereby reducing the net steering effect. The $E y$ field profile of QWR with $\beta_{d}=0.15$ and the calculated steering kick for the fifth focusing periods are shown in Fig. 11. The steering for the first focusing period is also shown in Fig. 11 for quantitative comparison. It can be seen that the introduction of the tilt angle helps in reducing the net kick to a value less than 0.2 mrad.

\section{RESULTS}

After carrying out the beam dynamics and steering calculations of the individual focusing periods, the entire beam line from 1.3 to $7.16 \mathrm{MeV} / \mathrm{u}$ has been simulated in GPT. Both transverse and longitudinal particle tracking have been performed. Proper phase and voltage multiplication values of 36 resonators used in GPT have been found using MATHEMATICA. The total length required to attain the final energy, keeping provisions for adequate distances in between the cryomodules, is around $18 \mathrm{~m}$. The longitudinal acceptance of the entire chain of 36 QWRs, calculated using GPT, is shown in Fig. 12. The final energy

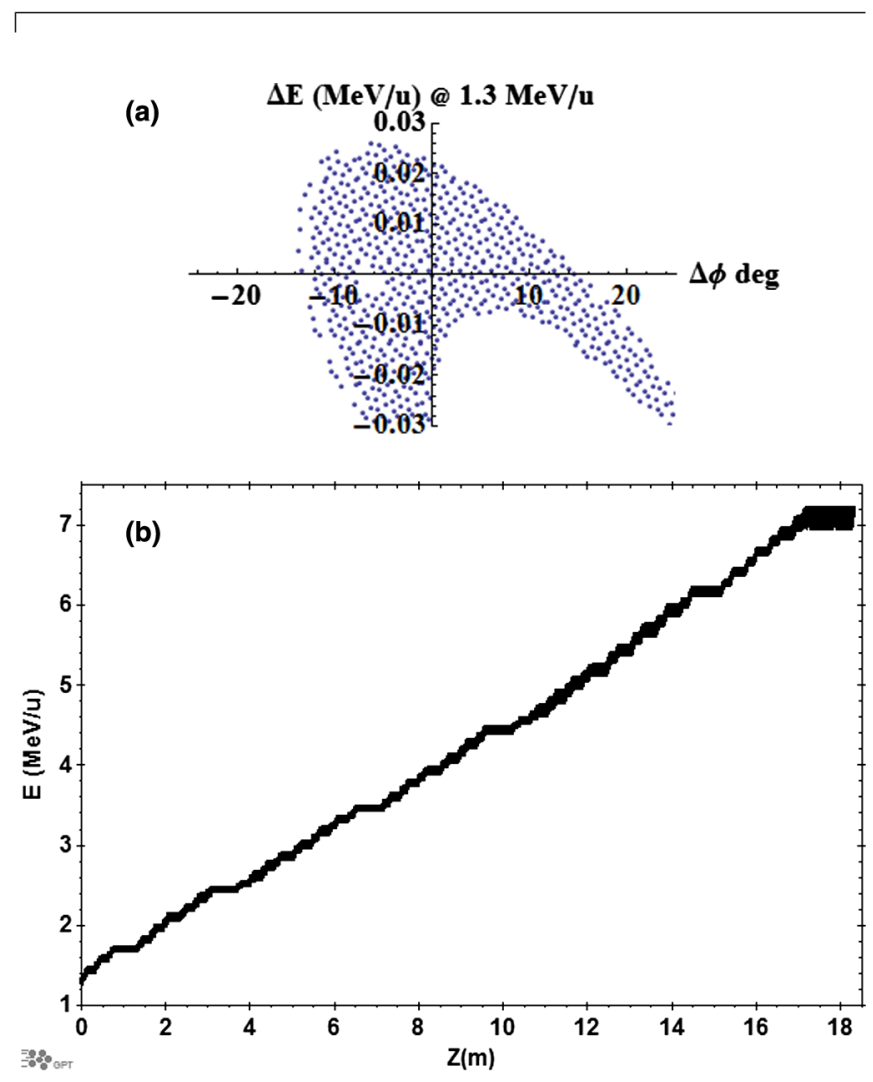

FIG. 12. (a) Longitudinal acceptance ellipse of entire beam line consisting of 36 resonators. (b) Energy variation along the length. 

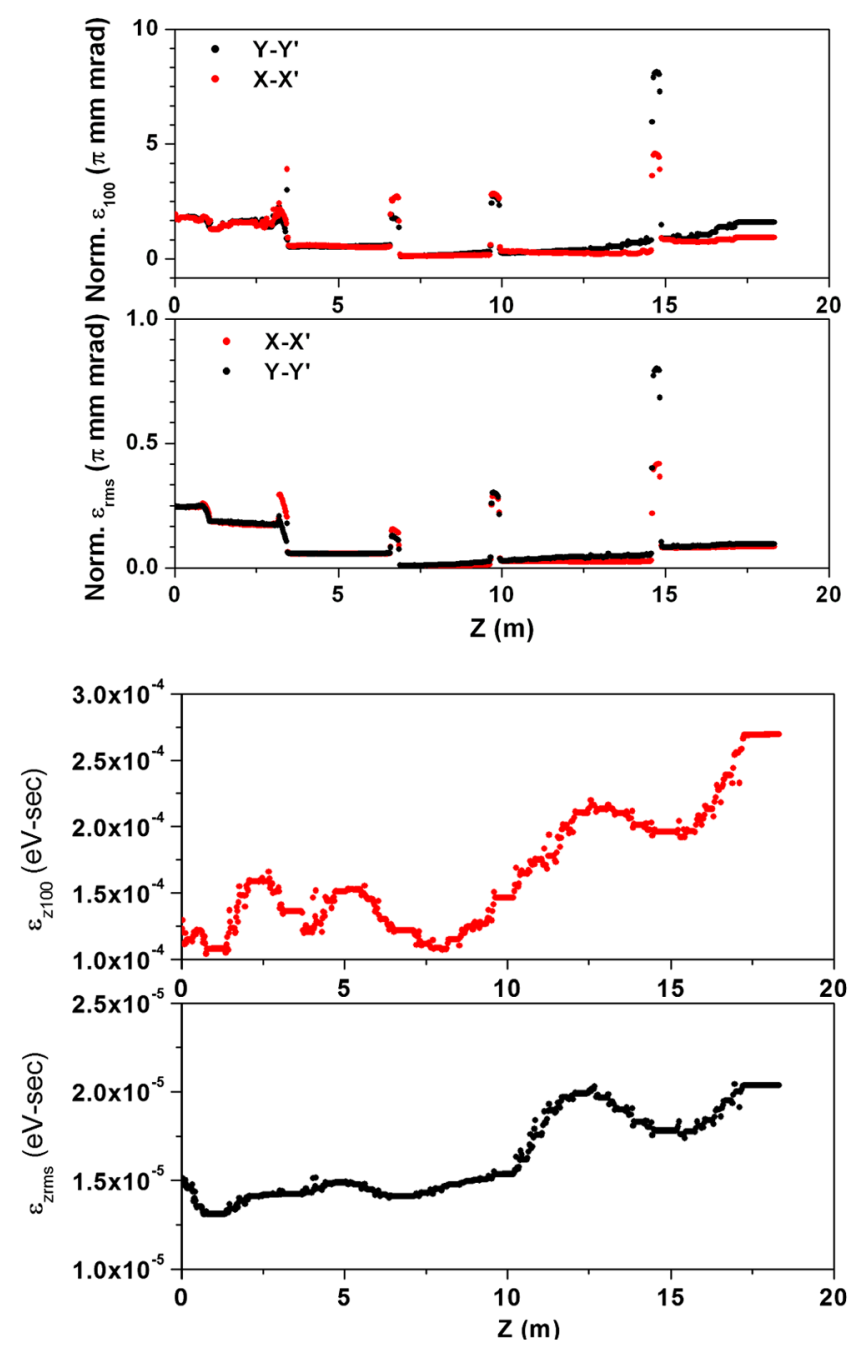

FIG. 13. Normalized longitudinal and transverse emittance value along the length.

of the accepted particles lies in between 7.00 to $7.20 \mathrm{MeV} / \mathrm{u}$. The energy variation along the length for the particles lying in the acceptance ellipse is also shown in Fig. 12.

In order to have an idea of emittance variation both in the $X$ and the $Y$ direction along the length, particle tracking of an ensemble with longitudinal distribution equivalent to acceptance ellipse (as shown in Fig. 12) has been carried out. The solenoids would provide an additional focusing for the beam. Since the transverse focusing depends critically on the rf phase seen by the particle, the focusing would be weaker as compared to the synchronous particle in the case of a particle with large phase offset. This is also in tune with the case when we operate the resonators at phase, different from the calculated value. With the beam having a longitudinal profile of phase width more than \pm 10 degree and energy width $\sim \pm 0.03 \mathrm{MeV} / \mathrm{u}$ (Fig. 12), we have used five solenoids (each of length $250 \mathrm{~mm}$ ) placed in each of the cryomodules in order to have the maximum beam radius less than $\pm 8 \mathrm{~mm}$ along the
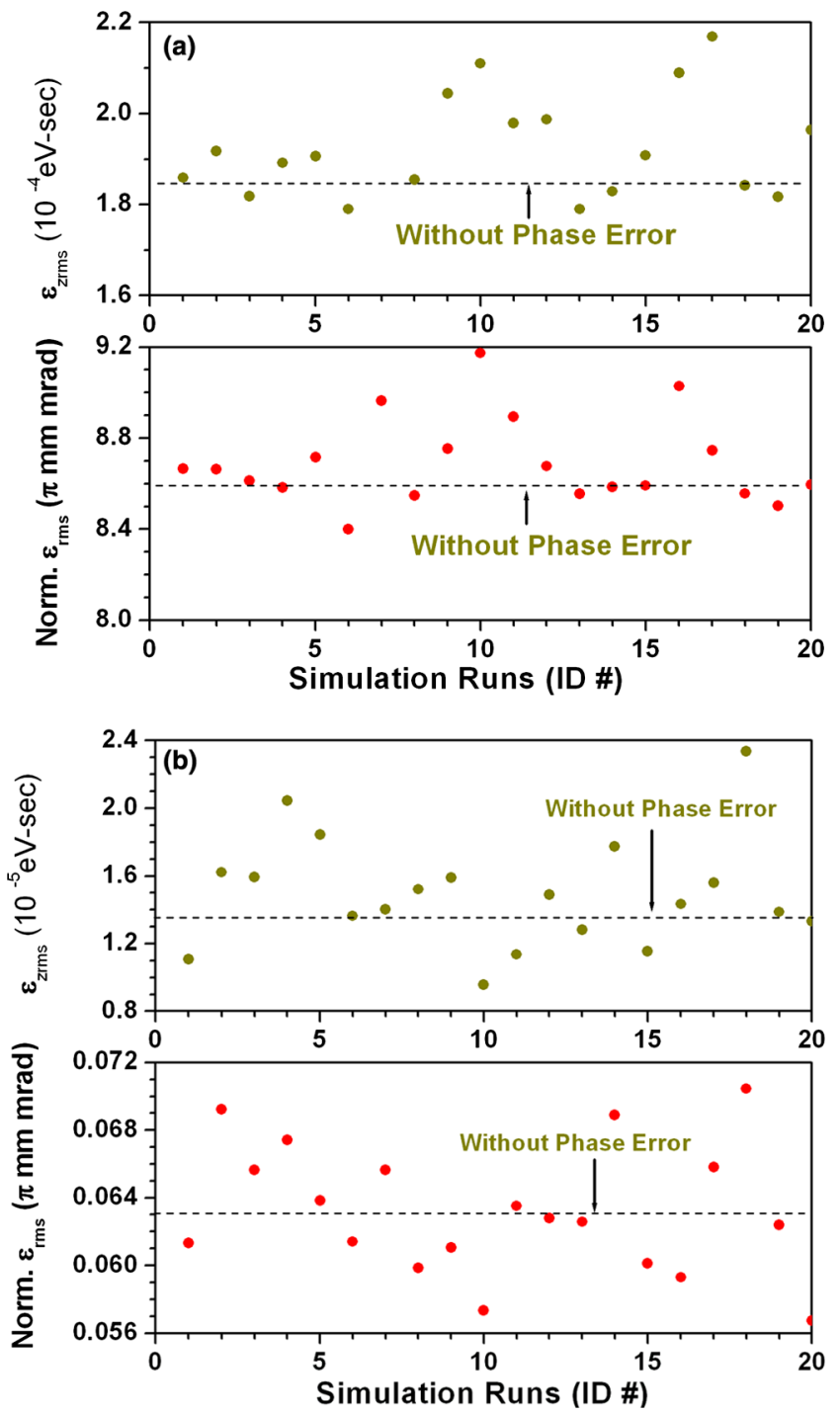

FIG. 14. Variation of longitudinal and transverse emittance with random phase error introduced in all the resonators over a range of (a) \pm 0.3 deg with input longitudinal beam having phase width $\pm 10 \mathrm{deg}$, and (b) $\pm 1 \mathrm{deg}$ with input longitudinal beam having phase width $\pm 5 \mathrm{deg}$.

entire beam line. Apart from the solenoid in the second cryomodule which needs $7.5 \mathrm{~T}$, all others need $6 \mathrm{~T}$. GPT calculated emittance values have been plotted in Fig. 13. The routine for calculation of rms emittance in GPT, in fact normalizes the emittance by multiplying the average gamma $(\gamma)$ factor, while emittance100 gives the area of the ellipse enclosing all the particles. In all the graphs shown in Fig. 13, the final normalized transverse ellipse does not show appreciable growth in emittance as compared to the initial value.

In the A-APF structure, described above, both the transverse and longitudinal focusing depends on the rf phase seen by the particle. Thus, the phase errors/instability would surely affect both transverse and longitudinal beam dynamics. To see the effect of phase error we have 
carried out 20 GPT simulations with phase errors introduced into the 36 resonators, without changing the strength of solenoids. For this calculation, using MATHEMATICA, 36 random numbers have been generated in the range of $\pm 0.3 \mathrm{deg}$ and $\pm 1 \mathrm{deg}$. Twenty such sets have been generated each for $\pm 0.3 \mathrm{deg}$ and $\pm 1 \mathrm{deg}$. For transmitting the beam with longitudinal emittance ellipse the same as the acceptance ellipse (shown in Fig. 12), having phase width of around $\pm 10 \mathrm{deg}$ and energy width of $\pm 0.03 \mathrm{MeV} / \mathrm{u}$, with $100 \%$ efficiency, a phase stability of $\pm 0.3 \mathrm{deg}$ is required. However, if the longitudinal emittance is reduced, having phase width of $\pm 5 \mathrm{deg}$, keeping the energy width the same, $100 \%$ transmission efficiency can be achieved with a phase stability of $\pm 1 \mathrm{deg}$. The output longitudinal and transverse rms emittance for both cases have been plotted in Fig. 14 with the dashed line showing the emittance value with the resonators tuned to the correct phases.

\section{CONCLUSION}

We have presented here the design of a long superconducting linac booster in the A-APF configuration capable of accelerating heavy ions of $q / A \geq 1 / 8$ from 1.3 to $7.16 \mathrm{MeV} / \mathrm{u}$. This configuration has allowed appreciable longitudinal acceptance and provides adequate focusing in both longitudinal and transverse directions. The A-APF configuration in our design comprises five focusing periods and QWRs designed for three beta values of $\beta_{d}$ equal to $0.06,0.1$, and 0.15 . The phase oscillation parameters have been fixed with the help of Smith-Gluckstern stability diagrams. A smooth approximation with acceleration has been used to calculate rf bucket size for each period. A particle tracking method has been utilized to recheck the phase acceptance of the periods. A steering effect analysis showed another inherent advantage of the present A-APF structure. The vertical kick in a particular direction is reduced owing to change in the sign of the synchronous phase of the resonators in a period. The entire accelerating structure from 1.3 to $7.16 \mathrm{MeV} / \mathrm{u}$ consists of 36 resonators spread over a length of $18 \mathrm{~m}$. The solenoid in each cryomodule can be tweaked to have control over the transverse beam profile. The appreciable longitudinal acceptance and adequate transverse focusing of the structure makes it suitable for accelerating rare ion beams with maximum efficiency. Since transverse and longitudinal optics depends entirely on the phase, phase errors and stability of the resonators have an effect on the beam dynamics.

\section{ACKNOWLEDGMENTS}

This work is a part of the research project funded by the Department of Atomic Energy for the development of a Rare Ion Beam Facility. The authors acknowledge Mr. H. K. Pandey for his kind help in CST simulation.

[1] M. L. Good, Phys. Rev. 92, 538 (1953).

[2] Y. B. Fayenberg, Zh. Tekh. Fiz. 29, 568 (1959).

[3] V. V. Kushin, "Accelerator with Alternating Phase Focusing," in Linear Ion Accelerators, edited by B.P. Murin (1978), Chap. 7, pp. 173-208 (in Russian).

[4] V. V. Kushin, in Proceedings of the 17th International Linear Accelerator Conference (LINAC94), Tsukuba, Japan, 1994 (KEK, Japan, 1994).

[5] K. W. Shepard, in Proceedings of the 10th Workshop on RF Superconductivity, Tsukuba, Ibakiri, Japan, 2001 (SRF, Tsukuba, 2001).

[6] S. A. Minaev, in Proceedings of the 2nd European Particle Accelerator Conference (EPAC 90), Nice, France, 1990.

[7] E. S. Masunov et al., in Proceedings of the 9th European Particle Accelerator Conference, Lucerne, 2004 (EPSAG, Lucerne, 2004).

[8] V. V. Kapin et al., in Proceedings of RuPAC 2010, Russia.

[9] A. Chakrabarti, Nucl. Instrum. Methods Phys. Res., Sect. B 261, 1018 (2007).

[10] R. K. Bhandari et al., in Proceedings of the 2nd International Particle Accelerator Conference, San Sebastián, Spain (EPS-AG, Spain, 2011).

[11] Computer Simulation Technology, Darmstadt, Germany [http://www.cst.com].

[12] General Particle Tracer [http://www.pulsar.nl/gpt].

[13] I. Ben-Zvi and J. M. Brennen, Nucl. Instrum. Methods Phys. Res. 212, 73 (1983).

[14] V. Kapin et al., Report No. HIMAC-075, 2003, p. 37.

[15] L. Smith and R. L. Gluckstern, Rev. Sci. Instrum. 26, 2201955.

[16] Wolfram Research, Inc., MATHEMATICA, Version 7.0, Champaign, IL (2008).

[17] H. Okamoto, Nucl. Instrum. Methods Phys. Res., Sect. A 284, 233 (1989).

[18] J. Qiang and R. W. Garnett, Nucl. Instrum. Methods Phys. Res., Sect. A 496, 33 (2003).

[19] B. Mustapha and P. Ostrumov, in Proceedings of the 25th International Linear Accelerator Conference LINAC10, Tsukuba, Japan (KEK, Tsukuba, Japan, 2010).

[20] A. Facco and V. Zvyaginstev, in Proceedings of the Particle Accelerator Conference, Chicago, IL, 2001 (IEEE, New York, 2001).

[21] P. N. Ostrumov and K. W. Shepherd, Phys. Rev. ST Accel. Beams 4, 110101 (2001).

[22] A. Facco and V. Zvyaginstev, Phys. Rev. ST Accel. Beams 14, 070101 (2011). 